\title{
Snapshot Navigation In The Wavelet Domain ${ }^{\star}$
}

\author{
Stefan Meyer ${ }^{1[0000-0002-3043-1683]}$, Thomas Nowotny ${ }^{1[0000-0002-4451-915 X]}$, \\ Paul Graham ${ }^{10000-0002-3745-0940]}$, Alex Dewar ${ }^{10000-0002-2432-5964]}$, and \\ Andrew Philippides ${ }^{1[0000-0001-5503-0467]}$ \\ University of Sussex, Falmer, Brighton BN1 9RH, UK
}

\begin{abstract}
Many animals rely on robust visual navigation which can be explained by snapshot models, where an agent is assumed to store egocentric panoramic images and subsequently use them to recover a heading by comparing current views to the stored snapshots. Long-range route navigation can also be explained by such models, by storing multiple snapshots along a training route and comparing the current image to these. For such models, memory capacity and comparison time increase dramatically with route length, rendering them unfeasible for smallbrained insects and low-power robots where computation and storage are limited. One way to reduce the requirements is to use a compressed image representation. Inspired by the filter bank-like arrangement of the visual system, we here investigate how a frequency-based image representation influences the performance of a typical snapshot model. By decomposing views into wavelet coefficients at different levels and orientations, we achieve a compressed visual representation that remains robust when used for navigation. Our results indicate that route following based on wavelet coefficients is not only possible but gives increased performance over a range of other models.
\end{abstract}

Keywords: Insect Navigation · Visual Homing · Wavelet.

\section{Introduction}

Many insects use view-based route following as part of their navigational toolkit $[35,11,13]$ and it is remarkable that with small brains and low resolution vision their visual route navigation is so efficient and robust [5]. The first computational model of insect visual navigation is the snapshot model [4]. This has inspired a series of view-based models. For example [2,3] have modelled how ants might store a set of views to guide them on long routes. Here, it is assumed that the ant stores snapshots experienced during route traversal. When lost, the ant recovers the right orientation by comparing rotated versions of its current view with all views in memory. The rotation that minimises the difference, or is the most familiar, yields the direction in which the ant should move $[36,2,3]$.

Animals or robots implementing view-based homing face challenges in the memory capacity needed to store views, as well as the computational power to

* This work was funded by the EPSRC (grant EP/P006094/1) and a University of Sussex scholarship. 
quickly compare them. For snapshot type models, the required memory increases with route length and so does comparison time [1]. Attempts to mitigate this effect have been made, by using different architectures of neural networks. One example would be the use of an infomax model $[2,15]$ to learn a familiarity function for each route. Instead of storing all views this model was trained with route views, such that it can output a familiarity value for any view, and use this for orientation recovery. Storing route views implicitly in an adjustable network like this allows for fixed memory size and comparison time up to a maximum of route length.

Another way of decreasing memory requirements is changing the representation of visual scenes. Many view-based models operate on pixel space $[8,36,32]$. However, due to its anatomy the insect visual system is highly unlikely to store and compare images in this way. Experimental evidence instead suggests that it implements a system of visual filters $[25,24,30]$, which process views to compressed representations formed by the filter outputs. Image differences can then be calculated in the co-domain of this new mapping. Typical examples of such a visual mapping include frequency based approaches like Fourier [6], Zernike [12] and Wavelet [16] transforms.

Fourier encoded views have been used in robotics in the context of place recognition in the past (e.g. [18]) and adapted for visual homing [31,27]. Extending on these findings, Zernike moments have been found to change between two locations in a way that allows to derive a homing direction $[29,33]$ and similar results have been reported for homing based on a subset of Haar wavelet responses [14].

Fourier transforms provide information about which frequencies occur in a signal, but do not convey any localisation of these frequencies (note, "localisation" here is used in accordance with the signal processing literature to denote the occurrence of a frequency in sample space and not the position of an agent). By contrast, the wavelet transform enables one to localise frequencies. This is done by convolving the input signal with discretely shifted and scaled versions of piecewise continuous functions called wavelets [16]. This property allows one to create a series of filters in a sub-band coding scheme [34], where each filter response yields a coefficient magnitude representing the occurrence of a certain frequency at a given location. Retaining the location of visual information is useful for navigation models (e.g. [23]).

To investigate frequency based image representations for route navigation, we trial a sparse wavelet based representation of views for route following. We chose filters corresponding to 'Haar' wavelets [10] which are plausible approximations to filters that are implemented in the visual system of an insect. Furthermore, they are well localised in space which for spatial tasks may be more important than precise information in the frequency domain. We compare the performance of wavelets, for both single and multi-snapshot navigation, to a series of pixelbased approaches including low-resolution features and skyline representations. We show that wavelets improve navigational performance suggesting promise for future, computationally efficient, navigation models. 


\section{Methods}

In order to investigate frequency based navigation, we utilised seven models of snapshot based route navigation, each of which operated on a different representation of images. The first stage of navigation is for the agent to traverse a pre-defined route (in ants this would be guided by path integration) during which the information needed for later visual navigation is stored. Here we collect images periodically along the route, and use these, after image processing, which is the key part of the investigation, as the stored snapshots.

Models: In order to retrieve a movement direction on a memorized route, a model $M$ maps an image $I_{Y}$ to $T_{Y}$ under different rotations $r$, compares it to a stored snapshot $T_{X}$, and returns the rotation $\hat{r}$ that minimises the Rotational Image Difference Function (RIDF) $\xi$ (similar to [36]). In this way a snapshot can be used to recall the direction the agent was facing when the snapshot was stored, also known as a visual compass. We measure performance by calculating the absolute angular error $\left|\hat{r}-r^{*}\right|$ between the angle $\hat{r}$ that minimises the RIDF and the true bearing $r^{*}$, which is known for each location. Small values correspond to small errors and can be interpreted as robust orientation recovery.

The most simple model $M_{p x}$ is directly operating in pixel space [2]. Comparison and storage are both realised in pixel space, such that the resulting RIDF is given by

$$
\xi_{p x}\left(I_{X}, I_{Y}, r\right)=\frac{1}{H * W} \sum_{h}^{H} \sum_{w}^{W}\left(I_{X}[w, h]-I_{Y}^{r}[w, h]\right)^{2}
$$

where $I_{x}[w, h]$ is a pixel value at width $w$ and height $h$ of the snapshot image, $I_{y}^{r}[w, h]$ is the pixel value of the current view rotated by $r, H$ is the number of rows and $W$ is the number of columns.

Next, we used three wavelet based models in order to analyse whether and how spatial localization of frequencies can be useful for orientation recovery. Specifically, we used the discrete wavelet transform (DWT) [26]. In order to perform a DWT on an image, it is treated as a $2 \mathrm{D}$ signal, where rows and columns are processed separately. Hence, high-pass filter responses at different orientations represent horizontal, vertical and diagonal details (edges), while the low-pass response remains an image approximation (blurred version of the original image). This approximation can be used as input for the next level of filter banks. Each of our three wavelet based models $M_{w v}^{L^{*}}$ extracts detail coefficients for each orientation (vertical, horizontal, diagonal) for a single level of interest $L^{*}$ by performing a Haar [10] wavelet based DWT on the image $I$ up to level $L^{*}$. It then omits all coefficients for which $L<L^{*}$ and sets the approximation matrix $c_{a}\left(L^{*}\right)$ to 0 . The remaining coefficients are then shaped into a representation matrix $C$ of size $P \times K$ where $P=\frac{H}{2^{L-1}}$ and $k=\frac{W}{2^{L-1}}$, consisting of 4 coefficient block matrices $0, c_{v}\left(L^{*}\right), c_{h}\left(L^{*}\right), c_{d}\left(L^{*}\right)$ of size $\frac{P}{2} \times \frac{K}{2}$ representing the orientational coefficients. 


$$
C\left(L^{*}\right)=\left[\begin{array}{cc}
0 & c_{v}\left(L^{*}\right) \\
c_{h}\left(L^{*}\right) & c_{d}\left(L^{*}\right)
\end{array}\right]
$$

For each block matrix, the $1 \%$ largest absolute coefficients are determined and the magnitude of the lowest of these is selected as a threshold: Every coefficient in the block that has smaller absolute value is set to zero. The resulting RIDF for any $L^{*}$ is then given as:

$$
\xi_{w v}\left(C_{X}, C_{Y}, r\right)=\frac{1}{P * K} \sum_{p}^{P} \sum_{k}^{K}\left(C_{X}[p, k]-C_{Y}^{r}[p, k]\right)
$$

where $C_{X}$ and $C_{Y}^{r}$ are the coefficient matrices for the view at the origin and the view at the displaced location with orientation $r$, respectively. It is worth mentioning that rotation is applied to the image before coefficient extraction. It can also easily be seen that $\xi_{w v}$ can be applied in an analogous way to each coefficient block matrix in an isolated fashion, which allows one to analyse the RIDF of each set of coefficients separately. In our experiments we used models with $\mathrm{L}=1, \mathrm{~L}=2$ and $\mathrm{L}=3$ (filter width 2, 4 and 8 pixel respectively) as models with higher values for $L$ performed poorly in pilot studies. The DWT is performed with MatLabs Wavelet Toolbox.

In order to get an understanding of how much accuracy is lost when discarding the approximation matrix, we additionally used a model $M_{\text {gauss }}$ that would use a Gaussian filter on an image with a filter width of $\sigma=5$ (determined experimentally), limiting the input signal to its lower frequency band. In addition, we compare our results to models which use the height of the skyline, a feature ants can use to navigate $[9,21]$, and which can be viewed as a low spatial frequency signal composed of oriented UV-contrast edges, which the ant visual system is tuned to $[20,19,22,27,28,7]$. Our final two models, therefore, consist of two approaches for extracting the skyline height in our virtual environment. $M_{s t}$ scans an image columnwise from the top until it encounters a pixel that is not sky coloured. The skyline value $S[w]$ at a specific image column $w$ is then given by the difference between height $H$ and the index $h$ of the encountered non-sky pixel. Repeating this procedure for each column of the image will result in a vector $S$ of size $1 \times W$. In an analogous way, $M_{s b}$ scans an image columnwise from the bottom up, until it encounters a pixel that is sky coloured. The resulting RIDF $\xi_{s}\left(S_{X}, S_{Y}\right)$ is given as the mean squared error between $S_{X}$ and $S_{Y}$

Simulated environment: AntWorld Experiments are conducted in a virtual environment (AntWorld, fig. 1), which was reconstructed from an ant field-site in Spain [17]. Lacking major landmarks, the world contains open areas filled with occasional and clumped grass tussocks (green patches in fig. 1). To compare different algorithm variants quickly and objectively, we use views that would be perceived by an agent from a $10 \mathrm{~cm}$ grid of positions (dots in fig. 1) for training and testing the navigation algorithms. These views are $360^{\circ}$ panoramic 


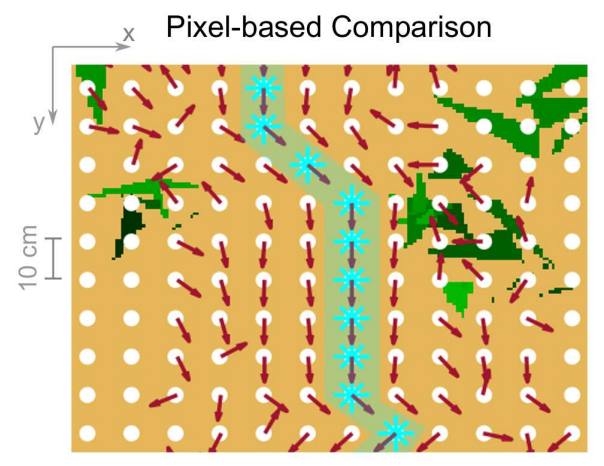

Wavelet-based Comparison

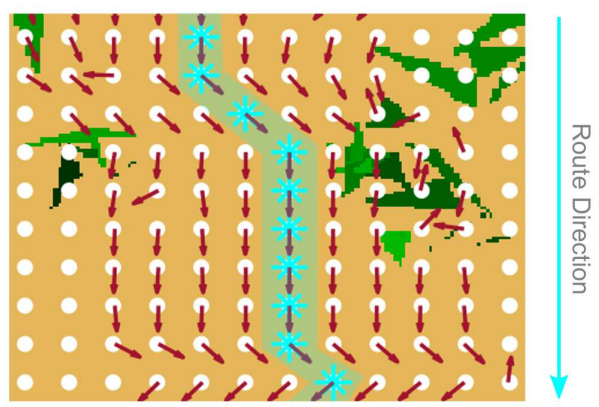

Fig. 1. Example route segments in the AntWorld. White dots show locations on the grid and turquoise dots are points on the route, in this case Route 2 (turquoise corridor). Each position in the vicinity of the route has a red arrow which shows the heading taken by an agent navigating by minimising the RIDF, using the respective view at the turquoise location as training data, with either unprocessed views (Pixel-based Comparison, left panel) or views encoded using wavelets (Wavelet-based Comparison, right panel). Green patches indicate vegetation from the AntWorld simulation.

greyscale images with a resolution of 720x150 pixels. For testing the algorithms, we generated 4 experimental routes consisting of a series of adjacent points on the grid (turquoise dots in fig. 1) with the bearing of the agent $r^{*}$ at each position defined as the direction from the current route point to the next one (red arrows in fig. 1). To test the algorithms, we used 8 laterally displaced copies of the route (each displaced by $10 \mathrm{~cm}$, up to $40 \mathrm{~cm}$ in both lateral directions, white dots in fig. 1) with the true bearing of these points set to the bearing of the nearest route point.

\section{Results}

RIDF: We first investigated how a wavelet based representation of visual input influences navigation in the AntWorld, starting by investigating the shape of the RIDFs when using a single snapshot. To do this we used a view at a typical location and calculated the RIDF $\xi_{p x}$ and $\xi_{w v}$ with nearby views. We then compared the heading recovered from the RIDF (i.e. the angle at which the minimum of the RIDFs occurred), $\hat{r}$, with the known target heading $r^{*}$. While the direction $\hat{r}_{p x}$ for the pixel based RIDF $\xi_{p x}$ diverges from the true bearing with increasing distance (fig. 2.a), the wavelet based RIDF $\xi_{w v}$ maintains a pronounced minimum close to $r^{*}$ (fig. 2.b) even when distant from the snapshot location.

Furthermore, we observed $\xi_{p x}$ to be smoother than any $\xi_{w v\left(L^{*}\right)}$. The calculation of the RIDF in pixel-space involves division by the number of pixels, therefore differences produced by displaced edges do not affect the overall shape much because large homogeneously coloured surfaces of objects raise no difference. On 

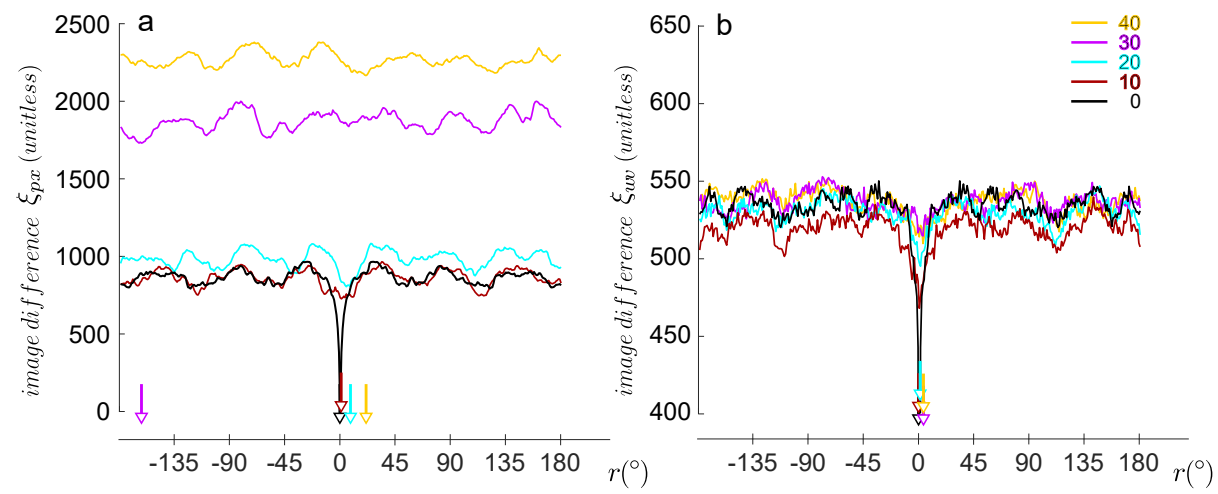

Fig. 2. Rotational Image Difference Functions for pixelwise and wavelet matching. RIDF $\xi$ for $0 \mathrm{~cm}$ (black), $10 \mathrm{~cm}$ (brown), $20 \mathrm{~cm}$ (turquoise), $30 \mathrm{~cm}$ (purple) and $40 \mathrm{~cm}$ (yellow) displacement from the route, for $M_{p x}$ (a) and $M_{w v}^{1}$ (b). Coloured arrows indicate the direction $r^{*}$ that minimises the respective RIDF.

the other hand, wavelet coefficients of level 1 represent the fine details results in large coefficients at edges of objects and not on smooth surfaces. Hence in a rotational movement, every edge contributes considerably to the RIDF, which leads to a more jagged appearance (fig 2. b).

Finally, we note that $\xi_{p x}$ saturates for bigger displacements, which likely is a property of our routes and environment. Routes are generated such that they remain in the open and do not cross patches of tussocks. Displacements however lead to views at locations that are close to tussocks. These tussocks occlude large amounts of sky, which increases the value of RIDFs.

In an attempt to further understand why we observe higher accuracy for $M_{w v}^{1}$, we selected multiple locations, where the model outperformed $M_{p x}$ in terms of angular error. Since the $\xi_{w v}$ is determined by the sum of the orientation specific filter responses, we focused on these first. In order to do so, we split $\xi_{w v}$ (shown in fig. 3.b) into one RIDF for each orientational filter response $c_{v}\left(L^{*}\right)$ (vertical), $c_{h}\left(L^{*}\right)$ (horizontal) and $c_{d}\left(L^{*}\right)$ (diagonal). In the following paragraph we will describe our results using a representative example location (view shown in fig.4) in order to visualise our findings in an instructive way.

We observed that the magnitude of the RIDF for each component differs (see fig. 3.a).

While the vertical coefficients have the highest magnitude, the shape of the overall RIDF showed a pronounced minimum at the correct rotation (fig. 3.a,red). Vertical coefficients correspond to vertical edges, which in the environment are related to borders of objects, which in the end determine the visual experience of any agent. Aligning vertical edges is hence similar to aligning objects, which is the underlying principle of visual navigation via landmarks. However, when using wavelet based RIDF it is not necessary to actually detect landmarks in two different views, match them and find the best alignment.Thus we assume 
that the existence of pronounced objects (like certain patches of tussocks) is the most relevant aspect of the environment determining the shape of the RIDF.

For horizontal features we observe multiple pronounced minima, one of which often occurs close to the true direction. However, the global minimum can often lead to erroneous orientation recovery (as seen in the example fig. 3.a, blue), when not paired with vertical components. Furthermore, we noticed that the difference between the rotation of the global minimum and the rotation of the next lowest local minimum is smaller for vertical than for horizontal features. In our environment, horizontal features correspond to the horizon line, and horizontal edges stemming from objects touching the ground. Hence the main factor that determines the shape of the RIDF is the distance of an object and the visibility of its base, as well as the visibility of the horizon in general.

Lastly, diagonal features produce a flat and noisy RIDF, that did not contribute to the location of the global minimum in a meaningful way (fig. 3.a, black).

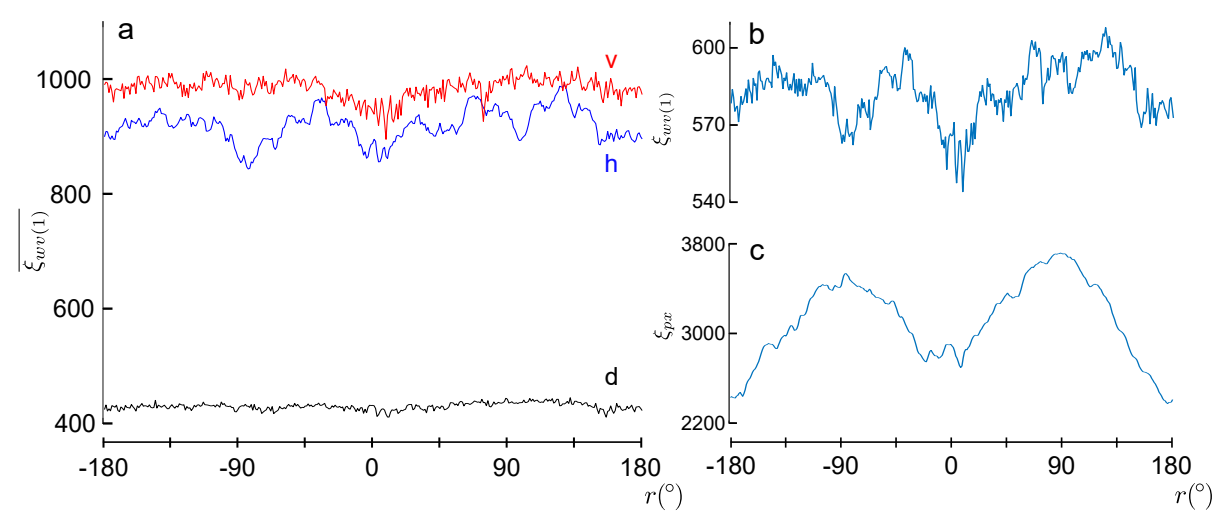

Fig. 3. Decomposed RIDF for separate wavelet components. (a) RIDF for vertical (red, v), horizontal (blue, h) and diagonal (black,d) for separate $M_{w v}^{1}$ components at an example position with 0 being the correct orientation. (b) combined RIDF $M_{w v}^{1}$ at the same position. (c) RIDF for pixel based model $M_{p x}$.

After investigating how $\xi_{w v}$ is shaped by its separate components, we next investigated how edge alignment yields more robust orientation recovery. Given our previous results, we focused on the vertical RIDF. We selected points on routes for which $\hat{r}_{p x}$ has been erroneous (absolute angular error bigger than 22.5) with regards to lateral displacement, but $\hat{r}_{w v}$ is not. In our experiments, we observed that these positions correspond to locations where displaced positions are in close proximity to tussocks that start to dominate the view (fig. 4.a.I and 4.a.II). If the object is not as prominent in the visual field at the snapshot location (fig. 4.a.I), the difference image (fig 4.a.IV) -and thus the RIDF- is dominated by the pixel difference introduced by the surface of such an object. Hence, $\hat{r}_{p x}$ under these circumstances is the heading that overlaps this object with another dense and homogeneously coloured structure (fig. 4.a.II and 4.a.V). However the main part 
of visual information is often not given by surfaces overlapping in colour, which leads to high angular errors. In contrast, vertical wavelet coefficients represent an image as the magnitude of vertical edges (fig. 4.b.I), which leads to a different effect. Instead of introducing an overrepresentation of pixels belonging to an object, an object in the wavelet domain results in a few more edges and possibly in the loss of some background edges due to occlusion (see fig. 4.b.II). While some edges are not visible any more, tussocks in medium proximity dominate the difference image (fig. 4.a.IV, tussock on the left) and thus the difference function due to their high spatial frequency. We believe this to be the explanation for the increased robustness of $M_{w v}^{1}$.

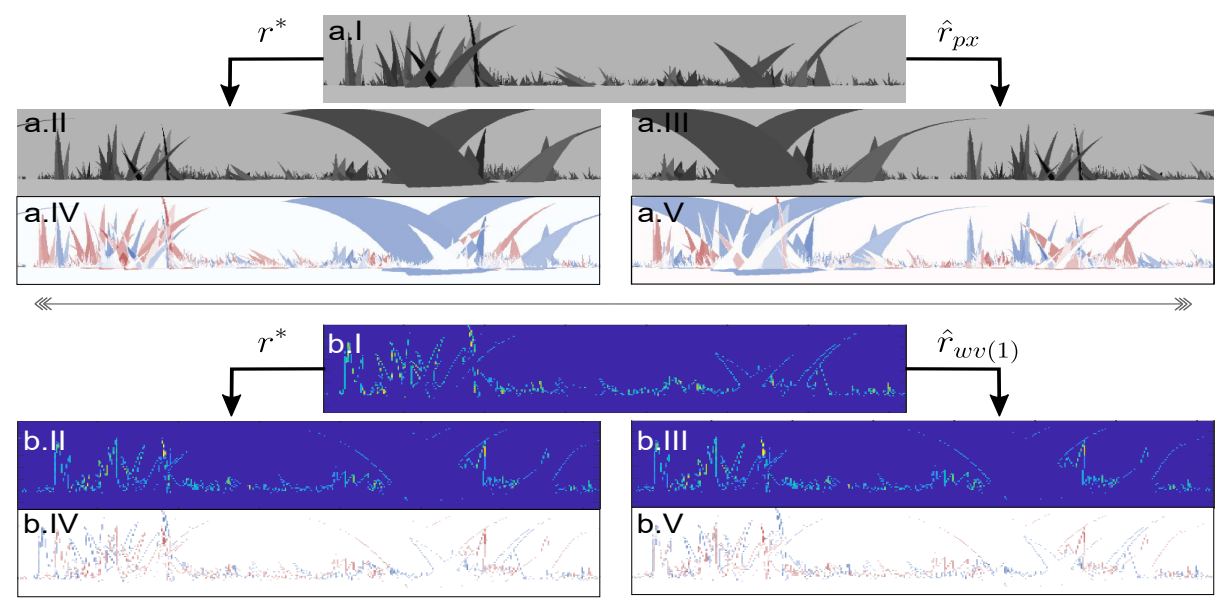

Fig. 4. Analysis of recovered headings for wavelet and pixelwise RIDFs. (a.I) snapshot at route location processed by $M_{p x}$. (a.II) View at displaced position $(30 \mathrm{~cm})$ in direction $r^{*}$, i.e. the correct direction(a.III) View at displaced position in direction $\hat{r}$, set by RIDF minimum with pixelwise matching (a.IV) Difference image between snapshot (a.I) and view with $r^{*}$ bearing (red colour = larger values in snapshot; blue colour $=$ larger values in view. (a.V) Difference image between snapshot and view with $\hat{r}_{p} x$ bearing. (b.I-b.IV) Analogous to (a) but processed by $M_{w v}^{1}$.

Angular Error Along Routes: In an attempt to connect our findings with regards to the RIDF to route following, we utilised 4 artificial routes. Each route is given by a main path, from which the snapshots are stored, and 4 lateral parallel paths on either side of the training route, spaced by $10 \mathrm{~cm}$, which comprise the test locations. For each test location, we calculated the bearing that results from comparison with the route snapshots and determined the angular error between $\hat{r}$ and $r^{*}$ for different models. In addition to the pixel space based model $M_{p x}$ and the wavelet coefficients of level 1 one model $M_{w v}^{1}$, we also included 5 other models. We used two more wavelet models based on coefficients of level $2\left(M_{w v}^{2}\right)$ and level $3\left(M_{w v}^{3}\right)$, as well as a model $M_{g}$ that performed a simple 


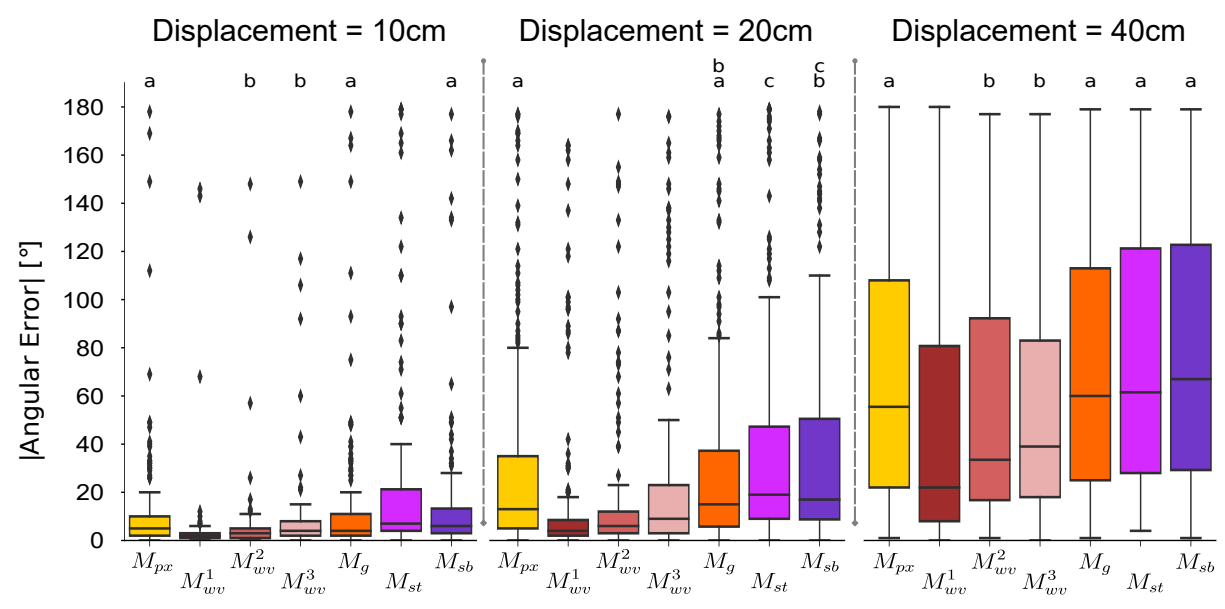

Fig. 5. Overall accuracy of heading recovery for different models. Angular error for different models (box labels) with increasing displacement (panels) from snapshot location as boxplots. Data is aggregated from 4 routes and displacements to either side of the route. In each panel, boxes sharing letters do not have significantly different medians. Boxes that do not share letters are significantly different ( $p$-value $<0.05$, Kruskal-Wallis test). $\mathrm{n}=139$ for each condition.

Gaussian low-pass filtering on images, to see how lower frequency components influence robustness. Furthermore, we used two different methods to extract the skyline height from images (scanning from top - $M_{s t}$ and scanning from bottom - $M_{s b}$ ) and calculated $\xi_{s t}$ and $\xi_{s b}$ from this one-dimensional input, to see how much information is provided by the shape of objects in front of the sky and the horizon.

For each channel and model, we collected the respective $\hat{r}$ and calculated the angular error at the 139 locations per channel. We then determined the median and the interquartile range (IQR) and visualised the distributions as boxplots (fig. 5). While IQR and median absolute angular error increased for each model with increased displacement from the route, the wavelet based models consistently show a lower IQR and median when compared to other models (fig. 5 , red boxes). Indeed, with increased limitation to the lower frequency band of the signal (as given by $M_{w v}^{2}, M_{w v}^{3}$ and $M_{g}$ ) we observe increased angular errors, indicating that high frequencies may carry important information about the environment. It is noteworthy however, that $M_{w v}^{2}$ performed similar to $M_{w v}^{1}$, offering the possibility of a simpler (lower resolution) option to decrease angular errors. Across all experiments we observe that skyline height based models $M_{s t}$ and $M_{s b}$ ) are not robust when compared to raw pixel space. This is a strong indication that the skyline height without further processing is not a robust feature for orientation recovery at least in this simulated world.

All models show a noticeable amount of data points outside the 1.5 IQR interval. Upon closer inspection of our environment and routes we have been able to identify different types of locations responsible for this. Quiver plots (see 
e.g. fig.1) reveal that most of these are in regions where paths cross through virtual tussocks, leading to strong occlusion effects which impair the validity of image based comparison for all models.

\section{Discussion}

Here we investigate how a wavelet based representation might be suitable for view based route navigation with the goal of increasing robustness against lateral displacements. We first observed how RIDFs at single points differ between pixel space and wavelet space. We found that RIDFs in wavelet space are more robust and we suggest that this is mainly due to vertical components of high level details. In order to connect our results with route following we then calculated angular errors along routes with lateral displacements and compared errors between a selection of wavelet- and pixel-based models. We have shown that the observed robustness of the wavelet model extends to route following and outperforms other models while increasing computational efficiency.

Stuerzl et al. [31] investigated how a Fourier coefficient representation of images influences homing performance. They found that frequency representations of images can be used effectively for snapshot-based visual homing (using the difference between Fourier components as a proxy for image difference). These results support the idea of using frequency components for visual navigation. However, a drawback of Fourier analysis is the inability to localise frequencies in an image, which makes it difficult to localise salient image components. An alternative to Fourier coefficients was introduced in [14], who extracted localised Haar-like image descriptors at random points in a snapshot and used responses to these filters to determine familiarity and ultimately derive a homing direction. In contrast, we used filter responses of one level of detail throughout and applied them to the whole image. Furthermore, our environment, though virtual, was adopted from a real ant site and is notably different from the toy world used by [31] and the office environment used by [31,14]. Similar to [29], who observed an increased catchment area when using Zernike Moments, we observed increased robustness against displacement from the original route when using wavelet coefficients. The core difference between their work and ours is that they used summed Zernike Moments, leading to a rotation invariant representation of an image, which wavelets are not. Thus, when compared to the results reported by $[31,14,29]$ for visual homing, our findings yield additional evidence that frequency based features are useful and in particular can be applied successfully to route following methods that rely on recovering headings.

In future work, we will optimise the wavelet approach for navigation in the real world. For instance, we intend to introduce a more elaborate mapping from wavelet coefficients to deriving a bearing and we will combine orientational features from different levels, which will add more energy from the lower frequencies which contain important information [31]. Finally, wavelets could be combined with an infomax learning rule [2] or a neural network (NN) trained to recall the route [23]. Alternatively, they could be used as the input to a biologically plau- 
sible network such as a mushroom body model [1]. As wavelets can be seen as analogous to the processing performed in the early stages of vision, optimising the wavelet coefficients or type for different NNs could not only improve navigation performance in robots but also shed light on aspects of the visual processing in insects.

\section{References}

1. Ardin, P., Peng, F., Mangan, M., Lagogiannis, K., Webb, B.: Using an insect mushroom body circuit to encode route memory in complex natural environments. PLOS Computational Biology 12(2), 1-22 (02 2016)

2. Baddeley, B., Graham, P., Husbands, P., Philippides, A.: A model of ant route navigation driven by scene familiarity. PLOS Computational Biology 8(1), 1-16 (01 2012)

3. Baddeley, B., Graham, P., Philippides, A., Husbands, P.: Holistic visual encoding of ant-like routes: Navigation without waypoints. Adaptive Behavior 19(1), 3-15 (2011)

4. Cartwright, B.A., Collett, T.S.: Landmark learning in bees. Journal of comparative physiology 151(4), 521-543 (Dec 1983)

5. Collett, T.S., Graham, P., Durier, V.: Route learning by insects. Current opinion in neurobiology 13(6), 718-725 (2003)

6. Cooley, J.W., Tukey, J.W.: An algorithm for the machine calculation of complex fourier series. Mathematics of computation 19(90), 297-301 (1965)

7. Differt, D., Möller, R.: Insect models of illumination-invariant skyline extraction from uv and green channels. Journal of Theoretical Biology 380, $444-462$ (2015)

8. Franz, M.O., Schölkopf, B., Mallot, H.A., Bülthoff, H.H.: Where did I take that snapshot? Scene-based homing by image matching. Biological Cybernetics 79(3), 191-202 (Oct 1998)

9. Graham, P., Cheng, K.: Ants use the panoramic skyline as a visual cue during navigation. Current Biology 19(20), R935-R937 (2009)

10. Haar, A.: Zur Theorie der orthogonalen Funktionensysteme. Georg-AugustUniversitat, Gottingen. (1909)

11. Harrison, J.F., Fewell, J.H., Stiller, T.M., Breed, M.D.: Effects of experience on use of orientation cues in the giant tropical ant. Animal behaviour (1989)

12. Khotanzad, A., Hong, Y.H.: Invariant image recognition by Zernike moments. IEEE Transactions on Pattern Analysis and Machine Intelligence 12(5), 489-497 (1990)

13. Klotz, J.: Topographic orientation in two species of ants (hymenoptera: Formicidae). Insectes sociaux 34(4), 236-251 (1987)

14. Lee, C., Kim, D.: Visual homing navigation with haar-like features in the snapshot. IEEE Access 6, 33666-33681 (2018)

15. Lulham, A., Bogacz, R., Vogt, S., Brown, M.W.: An infomax algorithm can perform both familiarity discrimination and feature extraction in a single network. Neural computation 23(4), 909-926 (2011)

16. Mallat, S.G.: A theory for multiresolution signal decomposition: the wavelet representation. IEEE transactions on pattern analysis and machine intelligence 11(7), 674-693 (1989)

17. Mangan, M., Webb, B.: Spontaneous formation of multiple routes in individual desert ants (cataglyphis velox). Behavioral Ecology 23(5), 944-954 (2012) 
18. Menegatti, E., Maeda, T., Ishiguro, H.: Image-based memory for robot navigation using properties of omnidirectional images. Robotics and Autonomous Systems $\mathbf{4 7}(4), 251-267$ (2004)

19. Möller, R.: Insects could exploit uv-green contrast for landmark navigation. Journal of theoretical biology 214(4), 619-631 (2002)

20. Mote, M.I., Wehner, R.: Functional characteristics of photoreceptors in the compound eye and ocellus of the desert ant, cataglyphis bicolor. Journal of comparative physiology 137(1), 63-71 (1980)

21. Müller, M.M., Bertrand, O.J.N., Differt, D., Egelhaaf, M.: The problem of home choice in skyline-based homing. PLOS ONE 13(3), 1-20 (03 2018)

22. Ogawa, Y., Falkowski, M., Narendra, A., Zeil, J., Hemmi, J.M.: Three spectrally distinct photoreceptors in diurnal and nocturnal australian ants. Proceedings of the Royal Society B: Biological Sciences 282(1808), 20150673 (2015)

23. Philippides, A., Graham, P., Baddeley, B., Husbands, P.: Using neural networks to understand the information that guides behavior: a case study in visual navigation. In: Artificial Neural Networks, pp. 227-244. Springer (2015)

24. Roper, M., Fernando, C., Chittka, L.: Insect bio-inspired neural network provides new evidence on how simple feature detectors can enable complex visual generalization and stimulus location invariance in the miniature brain of honeybees. PLoS computational biology 13(2) (2017)

25. Seelig, J.D., Jayaraman, V.: Neural dynamics for landmark orientation and angular path integration. Nature 521(7551), 186-191 (2015)

26. Shensa, M.J., et al.: The discrete wavelet transform: wedding the a trous and mallat algorithms. IEEE Transactions on signal processing 40(10), 2464-2482 (1992)

27. Stone, T., Differt, D., Milford, M., Webb, B.: Skyline-based localisation for aggressively manoeuvring robots using UV sensors and spherical harmonics. In: 2016 IEEE International Conference on Robotics and Automation (ICRA). pp. 5615$5622(2016)$

28. Stone, T., Mangan, M., Ardin, P., Webb, B.: Sky segmentation with ultraviolet images can be used for navigation. Berkeley, USA (2014), robotics: Science and Systems

29. Stone, T., Mangan, M., Wystrach, A., Webb, B.: Rotation invariant visual processing for spatial memory in insects. Interface Focus 8(4), 20180010 (2018)

30. Strother, J., Nern, A., Reiser, M.: Direct observation of on and off pathways in the drosophila visual system. Current Biology 24(9), 976 - 983 (2014)

31. Stuerzl, W., Mallot, H.: Efficient visual homing based on fourier transformed panoramic images. Robotics and Autonomous Systems 54(4), 300 - 313 (2006)

32. Stuerzl, W., Zeil, J.: Depth, contrast and view-based homing in outdoor scenes. Biological cybernetics 96(5), 519-531 (2007)

33. Sun, X., Yue, S., Mangan, M.: A decentralised neural model explaining optimal integration of navigational strategies in insects. eLife 9, e54026 (jun 2020)

34. Vetterli, M., Kovacevic, J.: Wavelets and subband coding. Prentice-hall PTR (1995)

35. Wehner, R., Michel, B., Antonsen, P.: Visual navigation in insects: coupling of egocentric and geocentric information. Journal of Experimental Biology 199(1), 129-140 (1996)

36. Zeil, J., Hofmann, M.I., Chahl, J.S.: Catchment areas of panoramic snapshots in outdoor scenes. J. Opt. Soc. Am. A 20(3), 450-469 (Mar 2003) 